\title{
Efeito da alteração do peso corporal sobre o desempenho reprodutivo de vacas leiteiras submetidas a diferentes níveis nutricionais no pré e no pós-parto Effect of Bodyweight Changes on Reproductive Perfomance in Dairy Cattle Cows under Two Levels of Nutrition during Pre-and Post-partum
}

\author{
Verônica Lobato ${ }^{1}$ e Ciro Alexandre Alves Torres ${ }^{2}$
}

\section{Resumo}

A alteração do peso corporal foi avaliada em 40 vacas de três graus de sangue, distribuídas em quatro tratamentos (T1, T2, T3 e T4), com variação no nível de suplementação concentrada nos períodos pré $(\mathrm{N}=$ normal e $A=$ alta) e pós-parto $(N 1=$ normal e $A 1+$ alta). Diferença significativa $(P<0,05)$ foi observada entre os graus de sangue em relação à alteração do peso corporal das fêmeas nos dias 30 e 60 do período pós-parto. Observou-se que animais com maior taxa de ganho de peso apresentaram menor intervalo, em dias, do parto à concepção: T2 = 83; T1 = 109,3; T4 = 114, T3 = 156,3.

Palavras-Chave: peso corporal, desempenho reprodutivo intervalo parto-concepção, fêmeas bovinas

\section{Introdução}

Face grande importância dos ruminantes na alimentação humana, torna-se imprescindível o controle dos fatores que interferem no rendimento dessas espécies. Por ser baixa a produtividade do rebanho nacional; justifica-se o estudo de técnicas que visem a sua melhoria. Como a alimentação é fator relevante, influindo no desempenho produtivo dos animais, deve-se maximizar os seus efeitos por meio de racionalização da sua utilização. Bovinos submetidos às condições tropicais, apresentam menores índices reprodutivos que os de clima temperado, sendo este parâmetro fortemente influenciado pela alimentação, pelo manejo reprodutivo e pelas condições climáticas.

É sabido que as deficiências nutricionais prejudicam todos os níveis de desenvolvimento dos animais e que a condição corporal, o peso, a alteração do peso no pós-parto, a estação do parto, a amamentação, a idade da mãe e a raça, são os principais fatores no alongamento do intervalo do parto à concepção. Estudos têm mostrado a importância do aumento da qualidade do alimento no final da gestação e/ou nas primeiras semanas após o parto, visto que ocorrem me-
Ihores resultados no desempenho reprodutivo, após o parto, quando se associa uma suplementação concentrada nos períodos pré e pós-parto.

Martinez et al. (1982) demonstraram que o peso corporal do parto, e a perda de peso durante os primeiros 90 dias de lactação, têm um efeito marcante no desempenho reprodutivo.

As vacas necessitam receber suplementação para evitar a perda de peso, e conseqüentemente o período de serviço.

As deficiências nutricionais, causadas pela qualidade e quantidade dos alimentos disponíveis para os animais, ocasionam perda acentuada de peso, ausência de estro, baixas taxas de concepção, longo intervalo parto-concepção e baixa produção de leite.

O desempenho reprodutivo das fêmeas bovinas está relacionados com o nível de energia ingerido antes e após o parto, e com aumentos na taxa de fertilidade dos animais submetidos à dieta com alto nível de energia que foram constatados por Wiltbank et al. (1964) e Dunn et al. (1969). Pleasants e Ginndza (1981) observaram que uma suplementação de alimentação concentrada 40 dias antes do parto não influenciou 0 período de anestro pós-parto. Wells et al. (1981) e Holness (1984) evidenciaram que a suplementação alimentar antes e após o parto, em pelo menos oito semanas, pode dar melhores resultados do que somente no período pós parto.

Imakava et al. (1987) demonstraram que a restrição energética tem ação direta no eixo hipotalâmicohipofisário, reduzindo a freqüência e a descarga de $\mathrm{GnRH}$, o que acarreta menor freqüência de pulso de $\mathrm{LH}$. Os níveis de progesterona ou $\mathrm{LH}$ plasmático em ciclos consecutivos de novilhas alimentadas não foram afetados pela restrição da dieta em 33\% do recomendado pelo NRC (Spitzer et al., 1978). Os autores afirmam, ainda, que nenhuma relação pode ser estabelecida entre alteração de peso e nível de progesterona, citando que a restrição energética não al-

\footnotetext{
Parte da tese (VL) apresentada à Universidade Federal de Viçosa, para obtenção do grau de Mestre em Zootecnia na área de Fisiologia Animal.

Professora Assistente, Departamento de Ciência dos Alimentos da Universidade Federal de Lavras, cp 37, 37.200-000, Lavras, MG, Brasil.

${ }^{2}$ Professor Titular, Departamento de Zootecnia, Universidade Federal de Viçosa, 36.500-000, Viçosa, MG, Brasil
} 
tera significativamente a fertilização, mas influencia os fatores que atuam mais tardiamente, causando morte embrionária.

Há grande evidência de que a condição corporal do parto tem importante efeito no reinício da atividade ovariana no pós-parto (Galina e Arthur, 1989). Folman et al. (1973) registraram que o plano nutricional e a alteração no peso corporal afetam vários fenômenos reprodutivos, e que têm efeitos significativo em vários fenômenos reprodutivos. O baixo nível nutricional prolonga o intervalo do parto à concepção, reduz as concentrações de progesterona e aumenta o número de inseminações requeridas por concepção. Uma correlação de $r=0,49(P<0,01)$ foi determinada entre o ganho de peso e o nível de progesterona do ciclo precedente ao da concepção, corroborando com os achados de Bogin et al. (1982), que observaram uma relação positivo entre ganho de peso no pós-parto e taxa de concepção.

Segundo Broster e Broster (1984) um bom plano nutricional no início da lactação beneficia o peso corporal e altos níveis de alimentação no período pré-parto estão associados com maior perda de peso no início da lactação. Shukla (1972) afirma que a composição da ração tem efeito na eficiência de conversão de energia da dieta para o tecido corporal. Reações com altos percentuais de forragem tendem a produzir mais ácido acético, o que acarreta menor eficiência em relação àquelas rações com alto percentual de concentrados, que aumentam a produção de ácido propiônico. Vacas apresentando ganho de peso antes e após o parto apresentam taxa de concepção de $80 \%$, enquanto naquelas que tiveram perda de peso em ambos os períodos, a taxa foi reduzida para $34 \%$. (Rodriguez e Parra, 1979).

\section{Material e Métodos}

O experimento foi conduzido no Setor de Bovinocultura do Departamento de Zootecnia do Centro de Ciências Agrárias da Universidade Federal de Viçosa em Minas Gerais.

Quarenta vacas mestiças holandesas, diagnosticadas como gestantes, foram alocadas em grupos de acordo com o grau de sangue (holandês puro por cruza = HPC, $9 / 16 \mathrm{HZ}$ e $7 / 8 \mathrm{HZ}$ ) e de peso vivo, de modo que os quatro tratamentos ficassem equilibrados quanto a esses dois fatores. Os animais foram submetidos a dois níveis nutricionais do $250^{\circ}$ dia de gestação até o parto, período pré-parto, e do parto à inseminação artificial, período pós-parto. Os animais foram distribuídos, aleatoriamente, em quatro tratamentos com dez vacas cada, assim caracterizados no período: $\mathrm{T} 1=\mathrm{A} ; \mathrm{T} 2=\mathrm{N} ; \mathrm{T} 3=\mathrm{A}$ e $\mathrm{T} 4=\mathrm{N}$, e no período pós-parto: $\mathrm{T} 1=\mathrm{N} 1 ; \mathrm{T} 2=\mathrm{A} 1 ; \mathrm{T} 3=\mathrm{A} 1$ e $\mathrm{T} 4=\mathrm{N} 1$, onde $\mathrm{N}$ corresponde a $2 \mathrm{~kg}$ de ração concentrada no período pré-parto, A corresponde a $4 \mathrm{~kg}$ de ração concen- trada no período pré-parto, $\mathrm{N} 1$ corresponde a $1 \mathrm{~kg}$ de ração concentrada para cada $3 \mathrm{~kg}$ de leite produzido no período pós-parto e $\mathrm{A} 1$ corresponde a $1 \mathrm{~kg}$ de ração concentrada para cada $1,5 \mathrm{~kg}$ de leite produzido no periodo pós-parto. No T1 as vacas receberam $4 \mathrm{~kg}$ de ração concentrada no período pré-parto e $1 \mathrm{~kg}$ de ração concentrada para cada $3 \mathrm{~kg}$ de leite produzido no período pós-parto. No T2 elas receberam $2 \mathrm{~kg}$ de ração concentrada no período pré-parto e $1 \mathrm{~kg}$ de ração concentrada para cada $1,5 \mathrm{~kg}$ de leite produzido no período pós-parto. No T3 as vacas receberam $4 \mathrm{~kg}$ de ração concentrada no período pré-parto e $1 \mathrm{~kg}$ de ração concentrada para cada $1,5 \mathrm{~kg}$ de leite produzido no período pós-parto. No T4 receberam $2 \mathrm{~kg}$ de ração concentrada no período pré-parto e $1 \mathrm{~kg}$ de ração concentrada para cada $3 \mathrm{~kg}$ de leite produzido no período pós-parto. Do parto ao $21^{\circ}$ dia o fornecimento de silagem e ração concentrada foram ad libitum para todos os animais.

Ficaram, assim, resumidos os tratamentos alimentares:

$\begin{array}{cc} & \text { PRÉ-PARTO } \\ \text { T1 } & 4 \mathrm{~kg} \\ \text { T2 } & 2 \mathrm{~kg} \\ \text { T3 } & 4 \mathrm{~kg} \\ \text { T4 } & 2 \mathrm{~kg}\end{array}$

PÓS-PARTO

$1 \mathrm{~kg}: 3,0 \mathrm{~kg}$ de leite

$1 \mathrm{~kg}: 1,5 \mathrm{~kg}$ de leite

$1 \mathrm{~kg}: 1,5 \mathrm{~kg}$ de leite

$1 \mathrm{~kg}: 3,0 \mathrm{~kg}$ de leite

O período pré-parto teve início aos 250 dias de gestação até o parto e o período pós-parto aos 22 dias de lactação e terminou após a primeira inseminação.

Os animais experimentais recebiam ração concentrada, às 6 horas e as eventuais sobras eram pesadas e registradas às 7 horas e registradas. Logo a seguir recebiam a silagem de cana e capim napier, ad libitum. As quantidades oferecidas e as sobras eram pesadas diariamente. A composição percentual dos ingredientes do concentrado e as percentagens dos nutrientes do concentrado e da silagem são mostradas, respectivamente nas tabelas 1 e 2 . Após a ingestão dos alimentos era oferecida água até a segunda alimentação, que se iniciava por volta das 13 horas, com 0 mesmo procedimento. As vacas ficavam soltas no curral, sem alimentos, mas com oferta de água.

Tabela 1 - Composição percentual da ração concentrada fornecida durante o experimento

\begin{tabular}{lc}
\hline Ingredientes & Quantidade (\%) \\
\hline Farelo de soja & 36,00 \\
Fubá de milho & 45,98 \\
Farelo de algodão & 15,00 \\
Uréia & 1,00 \\
Farinha de osso & 1,00 \\
Sal Mineral & 1,00 \\
Premix* & 0,02 \\
\hline
\end{tabular}

* Sulfato de cobre; Sulfato de Ferro; Sulfato de Zinco; lodato de Potássio; Cobalto. 
Tabela 2 - Teores de matéria seca (MS), proteínas bruta $(P B)$, cálcio $(C A)$ e fósforo $(P)$ da ração concentrada e do volumoso

\begin{tabular}{lllll}
\hline Ingredientes & $\mathrm{MS}(\%)$ & $\mathrm{PB}(\%)$ & $\mathrm{Ca}(\%)$ & $\mathrm{P}(\%)$ \\
\hline $\begin{array}{l}\text { Silagem (cana }+23,61 \\
\text { capim napier) }\end{array}$ & ${ }^{\star} 5,20$ & ${ }^{\star} 0,39$ & ${ }^{\star} 0,05$ \\
$\begin{array}{l}\text { Concentrado } \\
86,58\end{array}$ & ${ }^{\star} 25,30$ & ${ }^{\star *} 0,41$ & ${ }^{\star \star} 0,37$ \\
\hline
\end{tabular}

* Percentagem na matéria seca

** Percentagem na matéria natural

Um dia após o parto tanto as vacas quanto as crias eram pesadas; as vacas continuaram, a ser pesadas semanalmente, até a inseminação artificial.

A análise utilizada foi a ANOVAG, que emprega 0 Método dos Quadrados Mínimos, no Sistema SAEG (Sistema de Análise Estatística e Genética) proposto por Euclides (1983), a fim de observar as diferenças nos parâmetros reprodutivos e produtivos, tendo como fonte de variação os tratamentos, graus de sangue e suas interações e covariando a ordem do parto.

Modelo: variável função tratamento grau de sangue interação tratamento *grau de sangue, covariando ordem do parto.

$\begin{array}{lc}\text { Fonte de variação } & \text { GL } \\ \text { Tratamento } & 3 \\ \text { Grau de sangue } & 2 \\ \text { Trat. X grau de sangue } & 6 \\ \text { Ordem de parto B linear } & 1 \\ \text { Resíduo } & 27\end{array}$

As médias foram analisadas pelo teste de Duncan. O coeficiente de correlação $(r)$ entre as variáveis foi de acordo com o teste de correlação de Pearson.

Os teores de matéria seca, proteína bruta, cálcio e fósforo da ração concentrada e da silagem foram determinados no Laboratório de Nutrição Animal (UFV), segundo métodos descritos por Silva (1990).

Foram feitas palpações retais, duas vezes por semana, para avaliação dos ovários e útero quanto ao desenvolvimento de folículos, de corpo lúteo e da involução uterina. A detecção dos estros das vacas foi feita por observação do comportamento e também com auxílio de uma fêmea androgenizada, portando um buçal marcador. Estas vacas foram inseminadas no segundo estro detectável pós-parto, com sêmen de comprovada fertilidade, sendo então excluídas do regime alimentar. O diagnóstico de gestação foi feito por palpação retal entre 40 e 60 dias após a inseminação.

\section{Resultados e Discussão}

Os dados de consumo diário médio são apresentados na Tabela 3. As diferenças $(P<0,01)$ ocorridas entre os tratamentos no consumo de ração concentrada, nos períodos pré e pós parto, foram em função do experimento, que visou variar a oferta a fim de analisar as diferentes respostas fisiológicas dos animais; aos níveis de ingestão. Em função destas diferenças, pode-se notar que a ingestão de matéria seca, proteína bruta, cálcio e fósforo diferiu $(P<0,01)$ entre os tratamentos (Tabelas 4 e 5). Nessas análises observa-se uma diferença no período pré e pós-parto no consumo de matéria seca, proteína bruta, cálcio e fósforo entre os tratamentos $(P<0,01)$ e matéria seca, proteína bruta, cálcio e fósforo pré-parto entre os graus de sangue $(P<0,05)$.

Em relação ao consumo de silagem não foram observadas diferenças significativas entre os tratamentos e os graus de sangue no período pós-parto.

Os pesos médios dos animais por tratamento e grau de sangue são mostrados nas Tabelas 6 e 7 respectivamente. $O$ peso vivo dos animais mostrou uma tendência a elevação quando da suplementação alta de concentrados no pós-parto. Os animais do T4 (NN1) que receberam suplementação de concentrado em nível normal, tanto no pré quanto no pós-parto, apresentaram uma tendência ao menor ganho de peso, a partir da segunda pesagem no período pré-parto. Estes animais e os do T1 apresentaram também um menor consumo de ração concentrada $(P<0,05)$ no pósparto (Tabela 3).

O ganho de peso no período pré-parto foi altamente correlacionado com o consumo pré-parto de concentrado $(r=0,51 ; P<0,01)$, de matéria seca $(r=0,47$; $P<0,01)$, com proteína bruta $(r=0,53 ; P<0,01)$, com cálcio $(R=0,51 ; P<0,01)$ e fósforo $(r=0,52 ; P<$ $0,01)$. Aos 30 dias pós-parto as vacas de maior grau de sangue zebuíno apresentaram maior ganho de peso (Tabela 7).

Os resultados do desempenho reprodutivo apresentaram correlação negativa com a alteração de peso entre o primeiro dia pós-parto e a última semana préparto: intervalo do parto ao desenvolvimento de folículos com cerca de $10 \mathrm{~mm}(r=-0,43 ; P<0,01)$, ao primeiro estro $(r=-0,26, P<0,05)$ e à concepção $(r=$ $-0,41, P<0,01)$. Selk et al. (1988) encontraram uma correlação de $r=-0,17$ entre o intervalo parto à concepção com a alteração do peso corporal logo após ao parto. Dias Gonzalez (1991) encontrou uma correlação de $r=-0,50,(P<0,05)$ entre a perda de peso corporal e o intervalo parto à concepção. Maior taxa de gestação e menor número de serviços por concepção foram encontrados em vacas com ganho de peso no período pós-parto, contrastando com aquelas que perderam peso, $61,5 \%$ e 1,63 vs. $47,2 \%$ e 2.11 , respectivamente (Youdan e King) (1977). 
No período pós-parto, observou-se que os animais que ganharam mais peso foram os que apresentaram menor intervalo parto à concepção e menor número de ciclos requeridos até a concepção (Tabela 8 ). Embora os animais do T3 também tivessem ganho peso significativo, neste período, esse ganho não refletiu positivamente no intervalo parto à concepção e no número de ciclos requeridos à concepção, talvez por influência de outros fatores.

As vacas do T2 apresentaram melhor performance reprodutiva e melhor índice de ganho de peso no período pós parto.

\section{Conclusão}

De acordo com os resultados do presente experimento, observou-se que o regime alimentar que associou altos níveis nutricionais pré e pós-parto não mostrou-se eficaz nas respostas reprodutivas, Além de ser mais dispendioso. Logo, recomenda-se a utilização de maior quantidade de suplementação alimentar no período pós-parto, período no qual os animais tendem a responder com uma maior perda de peso e melhores resultados reprodutivos.

\section{Abstratct}

\section{Effect of Bodyweight Changes on Reproductive Performance in Dairy Cattle Cows under Two Levels of Nutrition during Pre and Post-partum}

The bodyweight changes were observed in 40 breeding dairy cows distributed in 4 treatments ( $T 1$, T2, T3, and T4) with variation in the levels of concentrate supplementation in pre-partum: $\mathrm{N}=$ normal and $\mathrm{H}=$ high and post-partum: $\mathrm{N} 1=$ normal and $\mathrm{H} 1=$ high, where, $\mathrm{T} 1=\mathrm{HN} 1, \mathrm{~T} 2=\mathrm{NH} 1, \mathrm{~T} 3=\mathrm{HH} 1$ and $T 4=N N 1)$. Significative difference $(P<0,05)$ was observed between treatments in relation to changes of bodyweight between breeding dairy cows in the days 30 and 60 postpartum. It was observed that animals with major gain weight showed shorter interval between parturition and conception $(\mathrm{T} 2=83.0, \mathrm{~T} 1=109.3, \mathrm{~T} 4=$ 114.00 and T3 $=156.3$ ).

Key words: Bodyweight, Reproductive Performance, Interval between Parturition, Dairy Cows.

\section{Tabelas}

Tabela 3 - Consumo diário médio $(\mathrm{kg})$ de ração concentrada e volumoso nos períodos pré e pós-parto, das vacas por tratamento e grau de sangue

\begin{tabular}{|c|c|c|c|c|c|c|}
\hline \multirow{2}{*}{ Trat } & \multicolumn{2}{|c|}{ Pré } & \multicolumn{2}{|c|}{21 Dias Pós } & \multicolumn{2}{|c|}{ Pós } \\
\hline & Conc. & Vol. & Conc. & Vol. & Conc. & Vol. \\
\hline $\mathrm{T} 1(\mathrm{AN})$ & $4,0 \mathrm{~A}$ & 21,3 & 8,8 & 17,0 & $5,2 \mathrm{~B}$ & 23,2 \\
\hline T2 (NA) & $2,0 \mathrm{~B}$ & 20,3 & 9,0 & 15,4 & $9,9 \mathrm{~A}$ & 20,6 \\
\hline T3 (AA) & $4,0 \mathrm{~A}$ & 19,9 & 8,5 & 15,0 & $10,2 \mathrm{~A}$ & 20,7 \\
\hline T4 (NN) & $2,0 \mathrm{~B}$ & 21,0 & 8,8 & 15,0 & $5,7 \mathrm{~B}$ & 22,0 \\
\hline HPC & 3,0 & $21,9 a$ & 8,1 & 15,6 & 6,9 & 22,6 \\
\hline $9 / 16$ & 2,9 & $18,4 \mathrm{~b}$ & 9,0 & 15,5 & 7,9 & 21,2 \\
\hline $7 / 8$ & 3,0 & $21,0 a b$ & 9,7 & 15,7 & 9,2 & 20.3 \\
\hline
\end{tabular}

Médias seguidas por letras diferentes na mesma coluna, diferem entre si pelo teste de Duncan. Letras maiúsculas comparam efeito de tratamento $(P<0,01)$ e letras minúsculas comparam efeito de grau de sangue $(P<0,05)$.

Tabela 4 - Consumo médio $(\mathrm{kg})$ de matéria seca e proteína bruta nos períodos pré e pós-parto, das vacas por tratamento e grau de sangue

\begin{tabular}{|c|c|c|c|c|c|c|}
\hline \multirow{2}{*}{ Trat } & \multicolumn{3}{|c|}{ MS } & \multicolumn{3}{|c|}{ PB } \\
\hline & Pré & 21 & Pós & Pré & 21 & Pós \\
\hline $\mathrm{T} 1$ (AN) & $8,5 \mathrm{~A}$ & 11,6 & $09,9 \mathrm{~B}$ & $1,2 \mathrm{~A}$ & 2,4 & $1,6 \mathrm{~B}$ \\
\hline T2 (NA) & $6,6 \mathrm{~B}$ & 11,4 & $13,5 \mathrm{~A}$ & $0,7 \mathrm{~B}$ & 2,4 & $2,7 \mathrm{~A}$ \\
\hline T3 (AA) & $8,1 \mathrm{~A}$ & 10,9 & $13,7 \mathrm{~A}$ & $1,2 \mathrm{~A}$ & 2,3 & $2,8 \mathrm{~A}$ \\
\hline T4 (NN) & $6,7 \mathrm{~B}$ & 11,2 & $10,1 \mathrm{~B}$ & $0,7 \mathrm{~B}$ & 2,4 & $1,7 \mathrm{~B}$ \\
\hline HPC & $7,8 \mathrm{a}$ & 10,7 & 11,3 & $1,0 \mathrm{a}$ & 2,2 & 2,0 \\
\hline $9 / 16$ & $6,9 b$ & 11,5 & 11,8 & $0,9 \mathrm{~b}$ & 2,4 & 2,2 \\
\hline $7 / 8$ & $7,5 a b$ & 12,1 & 12,7 & $1,0 \mathrm{a}$ & 2,6 & 2,5 \\
\hline
\end{tabular}

Médias seguidas por letras diferentes na mesma coluna, diferem entre si pelo teste de Duncan. Letras maiúsculas comparam efeito de tratamento $(P<0,01) \mathrm{e}$ letras minúsculas comparam efeito de grau de sangue $(P<0,05)$. 
Tabela 5 - Consumo diário médio ( $\mathrm{g}$ ) de cálcio e fósforo nos períodos pré e pós-parto, das vacas por tratamento e grau de sangue

\begin{tabular}{|c|c|c|c|c|c|c|}
\hline \multirow{2}{*}{ Trat } & \multicolumn{3}{|c|}{ Cálcio } & \multicolumn{3}{|c|}{ Fósforo } \\
\hline & Pré & 21 & Pós & Pré & 21 & Pós \\
\hline T1 (AN) & $36,3 \mathrm{~A}$ & 57,7 & $43,0 \mathrm{~B}$ & $17,3 \mathrm{~A}$ & 33,7 & 22,2 B \\
\hline T2 (NA) & $27,5 \mathrm{~B}$ & 52,2 & $61,0 \mathrm{~A}$ & $09,8 \mathrm{~B}$ & 35,3 & $39,3 \mathrm{~A}$ \\
\hline T3 (AA) & $35,1 \mathrm{~A}$ & 49,7 & $62,1 \mathrm{~A}$ & $17,1 \mathrm{~A}$ & 33,3 & $40,2 \mathrm{~A}$ \\
\hline T4 (NN) & 27,7 B & 51,1 & 44,3 B & $09,8 \mathrm{~B}$ & 34,5 & $23,7 \mathrm{~B}$ \\
\hline HPC & $33,0 \mathrm{a}$ & 48,7 & 49,9 & $13,8 \mathrm{a}$ & 32,0 & 28,5 \\
\hline $9 / 16$ & $29,1 b$ & 52,3 & 52,8 & $12,9 b$ & 32,5 & 29,9 \\
\hline $7 / 8$ & $31,9 a b$ & 55,6 & 57,4 & $13,5 \mathrm{Aa}$ & 38,0 & 36,4 \\
\hline
\end{tabular}

Médias seguidas por letras diferentes na mesma coluna, diferem entre si pelo teste de Duncan. Letras maiúsculas comparam efeito de tratamento $(P<0,01) \mathrm{e}$ letras minúsculas comparam efeito de grau de sangue $(P<0,05)$.

Tabela 6 - Pesos vivos médios $(\mathrm{kg})$ das vacas, nos períodos pré e pós-parto, por tratamento

\begin{tabular}{lcccc}
\hline Período & T1 (AN) & T2 (NA) & T3(AA) & T4 (NN) \\
\hline Pré & & & & \\
30 & 484,7 & 500,9 & 497,2 & 497,2 \\
15 & $501,7 \mathrm{~A}$ & $506,8 \mathrm{AB}$ & $513,8 \mathrm{~A}$ & $501,2 \mathrm{~B}$ \\
07 & 509,7 & 509,3 & 517,5 & 500,0 \\
Pós & & & & \\
01 & 460,0 & 460,3 & 459,5 & 441,0 \\
07 & 453,5 & 454,5 & 454,5 & 435,5 \\
15 & 445,5 & 448,2 & 453,0 & 435,7 \\
30 & 444,7 & 458,2 & 457,2 & 440,2 \\
60 & 444,7 & 457,7 & 468,0 & 442,5 \\
90 & 459,7 & 484,0 & 478,5 & 452,8 \\
\hline
\end{tabular}

Médias seguidas por letras diferentes na mesma linha, diferem entre si pelo teste de Duncan. $(P<0,05)$.
Tabela 7 - Pesos vivos médios $(\mathrm{kg})$ das vacas, nos períodos pré e pós-parto, por grau de sangue

\begin{tabular}{llll}
\hline $\begin{array}{l}\text { Período } \\
\text { do Parto }\end{array}$ & HPC & $9 / 16$ & $7 / 8$ \\
\hline Pré & & & \\
30 & 482,3 & 509,1 & 503,2 \\
15 & 494,1 & 519,8 & 513,0 \\
07 & 497,3 & 524,2 & 515,8 \\
Pós & & & \\
01 & 439,8 & 476,0 & 462,2 \\
07 & 433,2 & 473,0 & 454,5 \\
15 & 428,0 & 468,4 & 454,0 \\
30 & $431,0 \mathrm{~B}$ & $471,1 \mathrm{~A}$ & $462,7 \mathrm{AB}$ \\
60 & $436,8 \mathrm{~B}$ & $479,6 \mathrm{~A}$ & $473,2 \mathrm{~A}$ \\
90 & 451,3 & 488,8 & \\
480,0 & & & \\
\hline
\end{tabular}

Média seguidas por letras diferentes na mesma linha diferem entre si pelo teste de Duncan $(P<0,05)$.

Tabela 8 - Intervalo do parto (dias), à presença de folículos ovarianos de $10 \mathrm{~mm}$ (IP-F), ao primeiro estro (IP-IE) à inseminação artificial (IP-IA), à concepção (IPC) e número de ciclo estrais à concepção (CE/C)

\begin{tabular}{lccccc}
\hline & IP-F & IP-19-Est & IP-IA & IP-Conc. & Ciclos/Conc \\
\hline T1 (AN) & 20,2 & 31,7 & 72,5 & $109,3 \mathrm{AB}$ & $4,2 \mathrm{AB}$ \\
T2 (NA) & 19,2 & 40,2 & 61,3 & $083,0 \mathrm{~B}$ & $2,5 \mathrm{~B}$ \\
T3 (AA) & 22,9 & 41,7 & 76,5 & $156,3 \mathrm{~A}$ & $5,2 \mathrm{~A}$ \\
T4 (NN) & 23,5 & 48,8 & 78,7 & $114,0 \mathrm{AB}$ & $3,0 \mathrm{~B}$ \\
HPC & $24,6 \mathrm{a}$ & $47,8 \mathrm{a}$ & 79,5 & 107,2 & 3,2 \\
9/16 & $16,0 \mathrm{~b}$ & 27,8 & 57,0 & 105,3 & 3,9 \\
7/8 & $23,8 \mathrm{a}$ & 40,8 ab & 75,2 & 143,1 & 4,7 \\
\hline
\end{tabular}

Médias seguidas por letras diferentes na mesma coluna, diferem entre si pelo teste de Duncan. Letras maiúsculas comparam efeito de tratamento e minúsculas efeito de grau de sangue $(P<0,05)$. 


\section{Referências Bibliográficas}

BOGIN, E., AVIDAR, Y., DAVIDSON, M., GORDIN S., ISRAELI, B. Effec of nutrition on fertility and blood composition in the milk cow. J. Dairy Res., v. 49, p. 13-23, 1982.

BROSTER, W.H. \& BROSTER, V. J. Reviews of the progress of dairy science: long term effects of plane of nutrition on the performance of the dairy cow. J. Dairy Res., v. 51, p. 149-196, 1984.

DIAS GONZALÉZ, F. H. Efeito da condição corporal de novilhas sobre a fertilidade, o perfil metabólico pós serviço e a sobrevivência embrionária. Viçosa, 1991. 118 p. Tese (Doutorado em Zootecnia) - Universidade Federal de Viçosa.

DUNN, T. G.; INGALLS, J.E.; ZIMMERMAN, P.R.; WILTBANK. J. N. Reproductive perfomance of 2 yerar-old hereford and angus heifers as influenced by pre and post-calving energy intake. J. Anim. Sci., v. 39, p. 719-723, 1969.

EUCLYDES, R.F. SAEG, Sistema de Análise Estatística e Genética. Viçosa: Universidade Federal de Viçosa, Central de processamento de Dados, 1983. $68 p$.

FOLMAN, Y., ROSENBERG, M., HERZ, Z., DAVIDSON ,$M$. The relationship between plasma progesterone concentration and conception in post-partum dairy cows maintained on two levels of nutrition. $J$. Reprod. Fert., v. 34, p.: 267-278, 1973.

GALINA, C.S. ARTHUR, G.H. Review of cattle reproduction in the tropics. Part. 3 Puerperium. Animal Breeding Abstracts. v. 57, n. 11, p. 899-910. 1989.

HOLNESS, D.H. The effects of pre-and-post partum levels of nutrition fertility cattle. Reproduction des ruminants en zone tropicale. In: RÉUNION INTERNATIONALE POINT-A-PITRE, Paris, Anais... INRA, 1984, p. 379-388.

IMAKAVA, K., DAY, M.L., ALESKY, D.D., CLUTTER. A., KITTOK, R.J., KINDER, J. E. Effects of 178 Estradiol and diets varying in energy on secretion of luteinizing hormone in beef heifers. J. Anim. Sci., v. 64, n. 3, p. 805-815, 1987.

MARTINEZ, N.D., VERDE, O., FENTON, F.R., LOPEZ, $S$. Liveweight change during the first 90 days of lactation and its effect on reproduction. Trop. Anim. Prod., v. 7, p. 105-112, 1982.
PLEASANTS, A.B., GININDZA, M.M. The effect of pré calving nutrition on the productivity of beef breeding cows in swaziland. Zimbabwe J. Agric. Res., v. 9, p. 7-12, 1981.

RODRIGUEZ, T.T., PARRA, N. Observaciones sobre 1 a fertilidad em vacas servidas al primer, segundo y tercer celo post-parto. Agron. Trop., v. 29, p. 251261, 1979.

SELK, G.E., WETTEMANN, R.P., LUSBY, K.S. Relationships among weight change, body condition and reproductive performance of range beef cows. J. Anim. Sci., v. 66, p. 3153-3159, 1988.

SHUKLA, K.S. Efficiency of utilization of energy and nitrogen for milk secretion by buffaloes fed various levels of concentrates. J. Dairy Res., v. 39, p. 421. 1972.

SILVA, D.J. Análise de alimentos (métodos químicos e biológicos). 2. ed. Viçosa, UFV, 1990, $165 \mathrm{p}$.

SPITZER, J.C., NISWENDER, G.D., SEIDEL, G.E., WILTBANK, J.N. Fertilizantion and blood levels of progesterone and $\mathrm{LH}$, in beef heifers on a restricted energy diet. J. Anim. Sci., v. 46, n. 4, p. 1071-1076, 1978.

WELLS, P.L., HOPLEY, J.D.H., HOLNESS, D.H. The influence of concentrate supplementation during the postpartum period on ovarian activity and conception. Ziwbabwe J. Agric. Res., v. 19, p. 1321, 1981 .

WILTBANK, J.N., ROWDEN, W.W., INGALLS, J.E., IMMERMAN, D.R. Influence of post-partum energy level on reproductive performance of hereford cows restricted in energy intake prior to calving. J. Anim. Sci., v. 23, n. 4, p. 1049-53, 1964.

YOUDAN, P.G., KING, O.L. The effect of bodyweight changes on fertility during the post-partum period in dairy cows. Brit. Vet. J., v. 133. p. 635-641, 1977. 